\title{
Percepção de Distância em Crianças durante a Locomoção
}

\author{
Eliane Mauerberg-deCastro ${ }^{12}$ \\ Renato Moraes \\ Universidade Estadual Paulista, Rio Claro
}

\begin{abstract}
Resumo
O objetivo do presente estudo foi investigar a percepção de crianças sobre distância na ausência de informação visual durante a locomoção. Ainda, se parâmetros biomecânicos relativos à locomoção são alterados durante a locomoção nas diferentes distâncias. Sete crianças na idade de 6 anos (GC) e 10 adultos (GA) foram convidados a andar vendados até alvos préestabelecidos. O expoente da função de potência e parâmetros biomecânicos (Ex.: duração da passada, proporção da passada e velocidade da passada) foram obtidos para cada grupo. Diferenças foram encontradas somente para os valores de distância produzida ao longo das distâncias testadas. Nenhum dos parâmetros biomecânicos diferiu entre GC e GA. Alterações quantitativas foram observadas nos parâmetros biomecânicos para distâncias curtas, embora o padrão do movimento não tenha sido alterado. Crianças desta faixa etária demonstram, em tarefas não usuais (Ex.: locomoção sem visão), adaptabilidade e capacidade de orientar-se no espaço utilizando apenas da sensibilidade háptica e provavelmente da imagem mental-construída da observação feita antes da realização da tarefa sem informação visual.

Palavras-chave: Psicofísica; biomecânica; percepção de distância; locomoção; desenvolvimento perceptivo.
\end{abstract}

\section{Distance Perception in Children while Walking}

\begin{abstract}
The purpose of this study was to investigate children's distance perception while walking blindfolded. Also, in order to measure differences at various distances, biomechanical parameters were assessed. Seven children, aged 6 (CG), and 10 adults (AG) were asked to walk to previously seen targets placed at different distances. Exponent and biomechanical parameters (i.e., stride duration, stride proportion, and stride velocity) were calculated for each group. The CG and AG exhibited similarities in biomechanical parameters, which for both groups changed with the length of the distance. We concluded that both children and adults used similar strategies to accomplish the distance perception task; that locomotion at short distances requires adaptations in gait patterns; and that children have a high capacity to adjust their behavior to unusual tasks (e.g., blindfolded locomotion).

Keywords: Psychophysics; biomechanics; distance perception; locomotion; developmental perception.
\end{abstract}

A percepção das propriedades espaciais pelos seres humanos é resultado de uma relação funcional específica entre organismo e ambiente que garante a evolução de comportamentos essenciais relativos à mobilidade, orientação e exploração. No início do processo de desenvolvimento, as crianças são aptas a obter informação através da visualização do ambiente e de objetos dentro deste, manipulação espontânea dos objetos e também através da locomoção. Como as crianças adquirem um amplo repertório locomotor (Ex.: engatinhar, andar e correr), elas adaptam dinamicamente seus processos perceptuais e aumentam sua capacidade exploratória. Estes processos perceptuais abrangem detalhes sobre

\footnotetext{
${ }^{1}$ Endereço para correspondência: Av. 24-A, 1515, 13506-900, Rio Claro, SP. Fone: (19) 526-4160/5264161/5264162/5264163. E-mail: mauerber@rc.unesp.br

${ }^{2}$ Os autores agradecem o apoio financeiro do CNPq (\#800827/91-0). Os autores agradecem também a colaboração de Juliana Schüller e Maricy Heintz durante a coleta e análise dos dados.
}

textura, forma, tamanho, direção, distância, orientação e a relação funcional entre sujeito e objeto.

$\mathrm{Na}$ área de psicofísica, um grande número de pesquisas sobre percepção de distância utilizam uma perspectiva "estática" para a medição dos sistemas sensoriais (DaSilva, 1983; DaSilva \& Macedo, 1983; Mauerberg, Schuller \& Moraes, 1996). Entretanto, a locomoção é um importante meio de obtenção de informação do ambiente e, em muitas situações diárias, a percepção de distância é utilizada numa perspectiva "dinâmica" (Ex.: andar na rua e estimar a distância até um ponto pré-estabelecido).

No campo da psicofísica sensorial e das medidas em torno da percepção, análises sobre percepção do espaço usualmente empregam funções psicofísicas e métodos estatísticos diversos. Estas ferramentas caracterizam uma abordagem importante no campo da psicometria pois permitem a mensuração dos mecanismos comportamentais subjacentes à sensação e percepção.

DaSilva (1983), utilizando-se da função psicofísica de potência apresentada por Stevens na década 1950 (para 
detalhes sobre a função psicofísica de potência veja S. S. Stevens, 1975), mostrou que a distância percebida aumenta proporcionalmente com a distância física. Esta proporção reflete-se nos valores do expoente psicofísico (i.e., da função psicofísica de potência).

Por várias décadas e em diversos estudos psicofísicos o expoente $(n)$ da função psicofísica de potência tem sido o principal parâmetro de análise dos diferentes sistemas sensoriais. On ne um parâmetro que reflete a inclinação da reta que relaciona o estímulo físico com a resposta subjetiva. Quando o $n$ é igual a 1, existe uma constância perfeita (ângulo de inclinação da reta igual a $45^{\circ}$ ), mas se o $n$ é maior do que 1 , existe uma tendência a superconstância (inclinação da reta maior do que $45^{\circ}$ ), e se o $n$ é menor do que 1 , existe uma tendência a subconstância (inclinação da reta menor do que $45^{\circ}$ ). DaSilva e Macedo (1983), a partir de uma extensa revisão da literatura, resumiram os expoentes de distância em valores em torno de 0,97 com um desvio-padrão de 0,22.

Segundo DaSilva, Santos e Silva (1983), a percepção de distância está sujeita à superconstância crescente conforme o desenvolvimento da criança. Eles testaram crianças de 5-7 a 9-10 anos e adultos numa tarefa de bissecção de distâncias. As crianças subestimaram as distâncias enquanto os adultos superestimam as distâncias. A mudança foi gradual com a idade. Ainda, o valor do expoente mostra sua dependência da amplitude dos estímulos. Para a amplitude de 1 a 6 metros o expoente médio é de 1,47, para a amplitude de 2 a 21 metros, 1,22 e de 2 a 180 metros, 1,16. Ou seja, quanto maior a amplitude menor o valor do expoente.

Muitos estudos sobre percepção de distância e orientação no espaço utilizam a locomoção sem o uso da visão-a principal fornecedora de estímulos do ambiente-para testar a coerência e a importância de sistemas perceptivos que complementam a função visual (Farrell \& Thomson, 1999; Rieser, Ashmead \& Taylor, 1990; Rieser \& Rider, 1991). Outro argumento é que, para deslocamentos curtos, a visão não é fundamental na tarefa uma vez que a ação em si exclui formas de atualização da posição e da direção, e depende mais do conhecimento prévio de onde se quer ir (Philbeck, Klatzky, Behrmann, Loomis \& Goodridge, 2001).

De fato, mesmo manipulando-se a velocidade do andar (Elliott, 1987), sujeitos normais estimam acuradamente as distâncias através da locomoção. Os resultados deste estudo mostram que atrasos no início da caminhada não têm impacto sobre a variabilidade na direção do movimento, porém a direção do movimento interage com a velocidade do andar. A variabilidade do andar em termos de direção depende somente da distância inicial do sujeito em relação ao alvo. Quanto mais longa a distância maiores são as possibilidades de mudança de direção.
Em um estudo recente (Mauerberg-deCastro \& cols., 2001), nós observamos que os erros de desvio na rota são mais evidentes do que os erros ou incoerência nos julgamentos de distância, embora estes últimos diminuam em magnitude estimada à medida que a dimensão física da distância aumenta. A produção de distância em tarefas de orientação por adultos portadores de retardo mental é realizada de forma similar à de adultos normais. Entretanto, o grupo portador de deficiência mental tende a cometer graves erros de orientação desviando-se da rota esperada. Nós atribuímos estas dificuldades espaciais aos problemas posturais típicos desta população (i.e., instabilidade na marcha, rigidez de passadas nas fases de amortecimento durante a locomoção, entre outros) e à falta de oportunidades de mobilidade e exploração do ambiente durante o processo de desenvolvimento.

Cohen, Hoffman, Kelley e Anday (1988), investigando a representação espacial de crianças em ambientes com barreiras explicam que as rotas devem ter uma relação funcional entre si. Aparentemente a estimação eficiente de distância está vinculada à coerência entre os alvos (i.e., rota funcional). Outros estudos têm se ocupado de restrições ambientais em torno da percepção visual de espaço e a capacidade de movimentação entre barreiras ao longo de trajetos (Corlett, Patla \& Williams, 1985; Okabe, Aoki \& Hamamoto, 1986; Warren \& Whang, 1987), aspectos da representação mental na estimação de distância durante a locomoção em adultos (Elliott, 1987; Corlett, Anton, Kozub \& Tardif, 1989; Rieser \& cols., 1990), crianças (Rieser, 1990) e outros poucos têm se ocupado da sensibilidade à estrutura espacial por indivíduos adultos mentalmente retardados (Mauerberg-deCastro \& cols., 2001; Rieser, Guth \& Weatherford, 1987).

Em geral estes estudos não têm se ocupado da análise de parâmetros da ação como fator de interferência em julgamentos perceptuais. Os parâmetros locomotores podem revelar estratégias de controle motor associadas à realização de tarefas psicofísicas. Por exemplo, Moraes (1999), ao avaliar a percepção de alturas de assentos por idosos, observou que os parâmetros de execução da tarefa (i.e., biomecânicos) não correspondem aos julgamentos de percepção de dificuldade ou facilidade das diferentes alturas. Ou seja, os idosos não discriminam as diferentes alturas de assentos como obstáculos ao conforto ou facilidade/dificuldade na execução do sentar, porém variáveis cinemáticas revelam instabilidade crescente no movimento, especialmente para as alturas mais baixas. Estes fatos denunciam um distanciamento na coerência entre percepção e ação pelo avanço da idade. 
A questão básica no presente estudo foi verificar em que medida resultados psicofísicos (julgamento de uma distância) podem refletir efeitos de restrições (privação de informação visual) sobre o controle da ação (alterações biomecânicas nos segmentos inferiores) de crianças comparadas com adultos. Assim, o objetivo do presente estudo foi investigar a organização da percepção de distância durante a locomoção em crianças na ausência de informação visual. Ainda, verificar a consistência de parâmetros biomecânicos nas diferentes distâncias produzidas.

\section{Método}

\section{Participantes}

Participaram do presente estudo 7 crianças com idade média de 6 anos $( \pm 0,58)$, recrutadas de uma colônia de férias promovida pela UNESP/Rio Claro para compor o grupo criança (GC). Dez adultos jovens com idade média de 22,4 anos $( \pm 2,27)$ foram recrutados do curso de educação física da UNESP/Rio Claro e compuseram o grupo adulto $(\mathrm{GA})$. As crianças foram autorizadas a participar do presente estudo por seus pais que assinaram um termo de consentimento. Os adultos jovens também assinaram um termo de consentimento aceitando as condições do estudo. Participantes em ambos os grupos informaram, diretamente (no caso dos adultos) ou através dos pais, não apresentar problemas de visão.

\section{Ambiente Experimental}

O ambiente experimental consistiu de um corredor delimitado por um sistema de roldanas com uma corda fina (referência tátil externa) onde foram realizados os deslocamentos durante a estimação de distância (Figura 1). O sistema de roldanas foi posicionado em uma área ampla e desimpedida de 20,0 x 7,23 metros excluídos os intervalos reservados antes do ponto de partida e após a distância

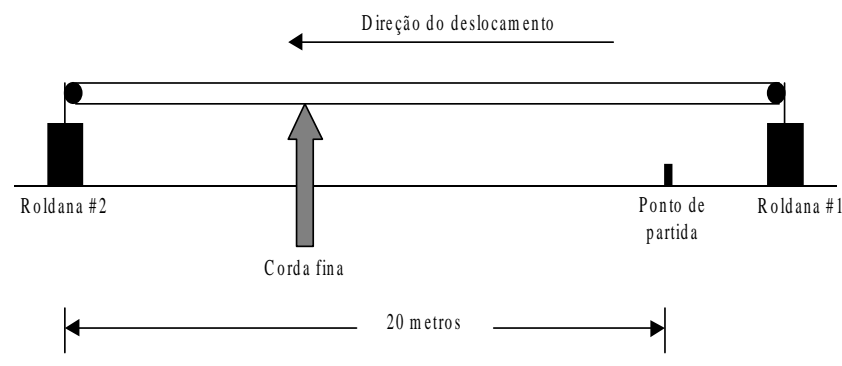

Figura 1. Representação esquemática do sistema de roldanas utilizado no presente estudo. máxima. O sistema de roldanas serviu para que o participante, tocando com uma das mãos, não desviasse da sua rota, aumentando a segurança durante a realização da tarefa.

Em todas as tentativas os participantes foram filmados no plano sagital esquerdo. Uma filmadora Sharp COMHQ (30 quadros por segundo) foi utilizada. Além disso, um sistema de referência $(1 \mathrm{x} 1 \mathrm{metro})$ foi utilizado para realizar a conversão para o tamanho real. A filmadora foi fixada à aproximadamente onze metros da área de percurso de modo a cobrir toda a sua extensão.

\section{Procedimentos}

Antes do início da tarefa psicofísica os participantes foram instruídos a realizar tentativas de prática andando em três situações diferentes: andar normal, andar com os olhos vendados sem referência e andar com os olhos vendados com o auxílio do sistema de roldanas. Nessa última condição os participantes puderam andar de uma extremidade à outra de modo a perceber a distância na qual o sistema de roldanas foi limitado. Em cada uma das condições de andar o participante praticou durante três minutos. As tentativas de prática foram dadas às crianças para que elas associassem a tarefa com um jogo. Nenhuma distância em particular foi treinada. A comparação entre as três formas de andar foi qualitativa e foi utilizada como critério de exclusão do participante. Ou seja, durante as práticas, caso algum participante apresentasse problemas de equilíbrio, de orientação de olhos abertos, ou de não entendimento da tarefa após extensa explicação o mesmo seria excluído. Nenhum dos participantes foi excluído em virtude dos fatores mencionados anteriormente.

No experimento, a tarefa dos participantes consistiu em andar vendado até um alvo (um brinquedo inflável de 50 $\mathrm{cm}$ de altura, conhecido pelas crianças como João Bobo) posicionado a uma distância previamente definida. As distâncias escolhidas foram de 2,00; 3,12; 5,10; 6,50; 8,30 e 9,95 metros. Os participantes foram instruídos a observar o objeto alvo durante aproximadamente cinco segundos. Após esse tempo os participantes foram vendados e solicitados a locomover-se em linha reta (utilizando o sistema de roldanas como referência) até onde o brinquedo foi colocado. Para evitar colisões, o brinquedo foi retirado e posicionado ao lado do trajeto para que o participante não tropeçasse enquanto ainda em deslocamento (Ex.: no caso da superestimação da distância). Duas séries de tentativas foram realizadas. Foi rigidamente controlada a possibilidade de contagem dos passos pelos participantes. Os mesmos foram convencidos de que a contagem atrapalharia o jogo. 
Os comprimentos da perna e da coxa foram obtidos no início de cada sessão experimental. Essas medidas foram utilizadas para a normalização das variáveis biomecânicas.

\section{Análise dos Dados}

Para a análise das filmagens foi utilizado um vídeo cassete de quatro cabeças com reprodução de imagem quadro a quadro marca Sony, uma placa de vídeo acoplada a um microcomputador 486DX-33. A placa de vídeo permitiu capturar a imagem através do computador e, utilizando uma rotina específica do software de imagem, foi possível obter as coordenadas x e y do calcanhar nos instantes que $o$ pé tocou o solo.

Com base nessas coordenadas foi possível calcular o comprimento da passada (CP). Uma passada foi definida como a distância entre dois eventos sucessivos do mesmo lado (Ex.: a passada iniciou com o toque do calcanhar do pé esquerdo no chão e terminou com o toque subseqüente do calcanhar do pé esquerdo no chão). O comprimento dos membros inferiores dos adultos $(0,81 \mathrm{~m})$ foi significativamente maior $(F(1,16)=96,898 ; p<0,01)$ do que o das crianças $(0,56 \mathrm{~m})$. Assim, para evitar comparações injustas entre adultos e crianças, o CP foi normalizado pelo comprimento do membro inferior (cmi) de modo a obter uma medida relativa, definida como proporção da passada (PP). Além dessa variável, a duração da passada (DP) foi obtida a partir da contagem do número de quadros do início ao término de cada passada. Esse número de quadros foi multiplicado pela duração de cada quadro $(0,033 \mathrm{~s})$ para obter a DP em segundos. Uma outra medida cinemática obtida foi a velocidade da passada (VP). A velocidade da passada foi obtida pela divisão da PP pela DP.

A média de cada variável biomecânica (PP, DP e VP) para cada estímulo em uma das séries de tentativas (definida por sorteio) foi analisada através de análise de variância para um fator (grupos) com medidas repetidas (seis estímulos). Para identificação das possíveis diferenças entre as medidas repetidas foram realizados testes $t$-student pareados com todas as combinações possíveis. O nível de significância adotado foi $p<0,01$, e o nível de $p<0,05$ como sendo marginal.

As distâncias produzidas pelos participantes foram medidas e esses valores foram analisados através de análise de variância para três fatores (2 grupos $\mathrm{x} 2$ séries de tentativas x 6 estímulos) com medidas repetidas nos dois últimos fatores (séries de tentativas e estímulos). Testes de Bonferroni ajustados para múltiplas comparações foram utilizados para identificar onde as diferenças residiram. Além disso, os dados foram analisados através da função de potência, usando para o cálculo do expoente e da constante escalar o método dos quadrados mínimos. Os valores do expoente foram submetidos a uma análise de variância para dois fatores (2 grupos x 2 séries de tentativas) com medidas repetidas no fator série de tentativas. Novamente testes de Bonferroni ajustados para múltiplas comparações foram utilizados para identificar onde as diferenças residiram. O nível de significância adotado foi o mesmo definido anteriormente. O coeficiente de determinação $\left(r^{2}\right)$ também foi calculado para verificar o nível de relacionamento entre estímulo (distância do objeto alvo até o ponto de partida) e resposta (distância produzida).

Com o objetivo de identificar diferenças nas tendências de respostas (sub, super ou constância perceptiva) uma análise de regressão linear foi realizada para cada participante em cada série de tentativa combinando estímulo e distância produzida. Posteriormente, para cada grupo, os valores da inclinação da reta e do intercepto foram comparados aos valores de um perfeito ajuste (i.e., 1 para inclinação da reta e 0 para o intercepto). Para tanto foram utilizados testes $t$ student para amostras independentes.

\section{Resultados}

\section{Parâmetros Biomecânicos}

A análise de variância para a PP não indicou efeito principal para os grupos testados. Entretanto, para a medida repetida estímulo houve efeito significativo marginal $(F(5,75)$ $=2,381, p<0,05)$. Através dos testes $t$-student foi possível identificar onde as diferenças ocorreram. A PP foi menor na distância de $2 \mathrm{~m}$ em comparação as distâncias de 5,10 m $(t(16)=-3,231, p<0,01), 6,50 \mathrm{~m}(t(16)=-3,055, p<0,01)$, $8,30 \mathrm{~m}(t(16)=-2,874, p<0,01)$ e $9,95 \mathrm{~m}(t(16)=-2,841, p$ $<0,01)$ (veja Figura 2a).

Para a DP também não houve efeito principal dos grupos testados. Para a medida repetida estímulo houve efeito significativo $(F(5,75)=5,877 ; p<0,01)$. Para o estímulo de $2 \mathrm{~m}$ a DP foi menor em comparação aos estímulos de 6,50 $\mathrm{m}(t(16)=2,608 ; p<0,01), 8,30 \mathrm{~m}(t(16)=2,755 ; p<0,01)$ e $9,95 \mathrm{~m}(t(16)=2,687 ; p<0,01)$. Para o estímulo de 3,12 $\mathrm{m}$, a DP também foi menor em relação aos estímulos de 6,50 $\mathrm{m}(t(16)=3,185 ; p<0,01), 8,30 \mathrm{~m}(t(16)=3,070 ; p<$ $0,01)$ e $9,95 \mathrm{~m}(t(16)=2,970 ; p<0,01)$ (veja Figura $2 \mathrm{~b}$ ).

Para a VP também não houve efeito principal dos grupos testados. Para a medida repetida estímulo houve efeito significativo $(F(5,75)=5,386, p<0,01)$. A VP na distância de $2 \mathrm{~m}$ foi menor em relação aos estímulos de $3,12 \mathrm{~m}$ $(t(16)=-2,217 ; p<0,05), 5,10 \mathrm{~m}(t(16)=-3,060 ; p<0,01)$, $6,50 \mathrm{~m}(t(16=-4,228 ; p<0,01), 8,30 \mathrm{~m}(t(16)=-3,582 ; p<$ $0,01)$ e $9,95 \mathrm{~m}(t(16)=-3,522 ; p<0,01)$. Houve também diferença entre os estímulos de 3,12 e 9,95 (t(16) = -2,505; $p$ $<0,05)$, sendo que a VP foi menor na distância mais curta (veja Figura 2c). 
a)

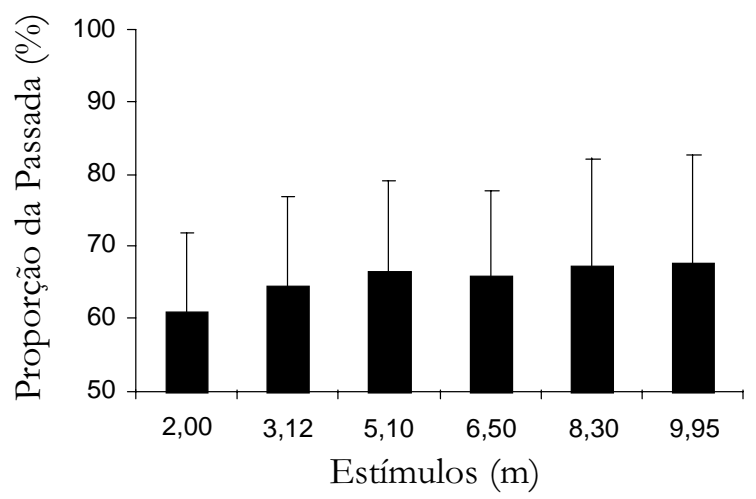

b)

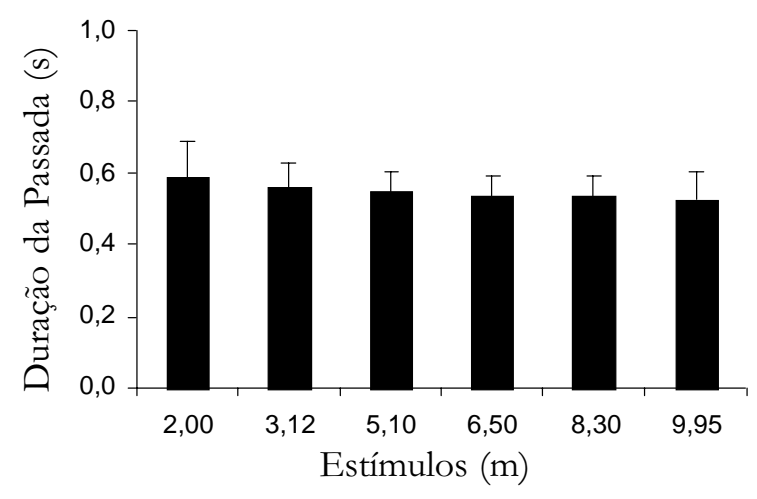

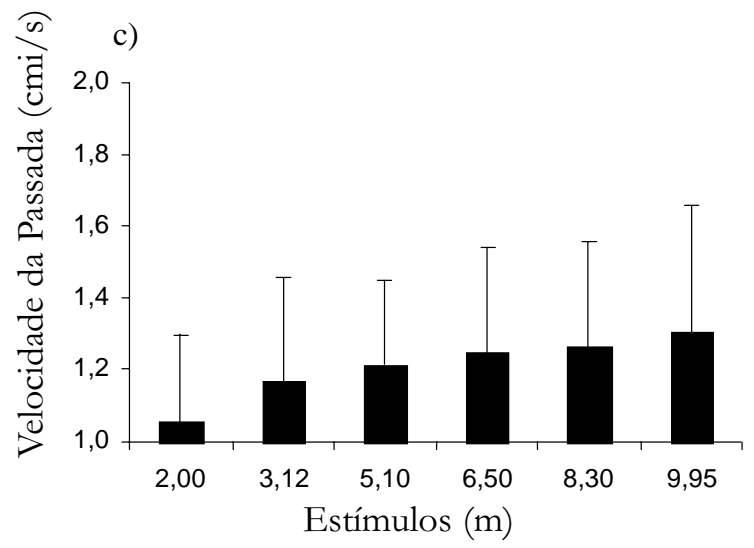

Figura 2. Média e desvio-padrão dos valores da proporção da passada (a), duração da passada (b) e velocidade da passada (c). Os valores da proporção da passada estão expressos como uma porcentagem do comprimento do membro inferior (cmi), enquanto a duração da passada está expressa em segundos (s) e a velocidade da passada em $\mathrm{cmi}$ por segundo ( $\mathrm{cmi} / \mathrm{s})$.

Parâmetros Psicofísicos

Não houve diferença significativa para os grupos testados em relação as distâncias produzidas. Da mesma forma, não houve diferença para a série de tentativas. Todavia, para a medida repetida estímulo houve efeito principal $(F(5,75)=$ 201,9; $p<0,01)$. O teste de Bonferroni ajustado para múltiplas comparações identificou diferenças entre todos os estímulos testados $(p<0,01)$. A interação estímulo com grupos foi marginalmente significativa $(F(5,75)=2,59 ; p<$ $0,05)$. Na Figura 3, podemos observar que o GA produziu maiores distâncias do que o GC nos três estímulos maiores $(6,50,8,30$ e $9,95 \mathrm{~m})$. A variabilidade no desempenho das crianças é levemente superior à dos adultos ao longo das distâncias.

A análise de variância para a variável expoente não identificou diferença entre os dois grupos testados nas duas séries de tentativas. Nas Tabelas 1 e 2 podemos

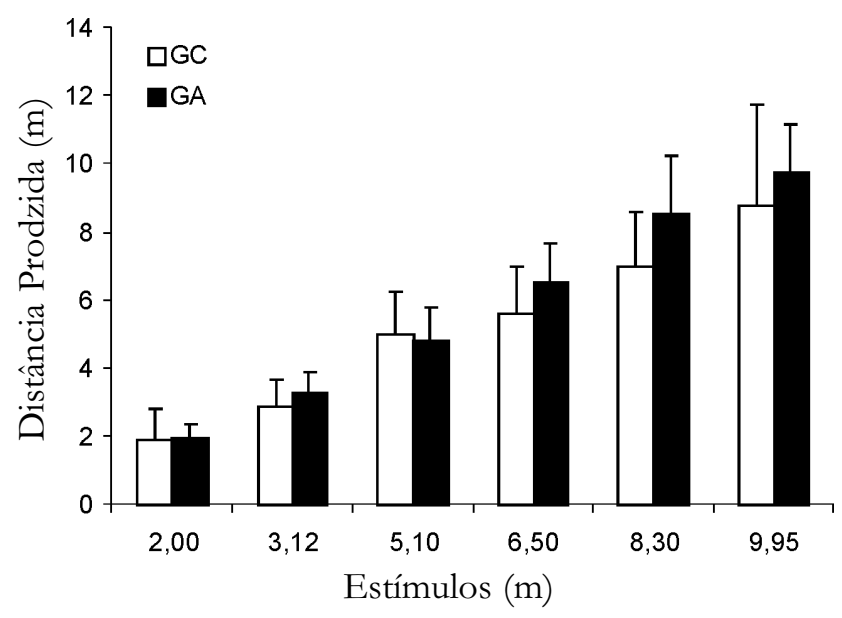

Figura 3. Média e desvio-padrão dos valores da distância produzida para os participantes do grupo criança (GC) e grupo adulto (GA). As colunas brancas representam o GC, enquanto que as colunas pretas o GA. 
Tabela 1

Valores dos Parâmetros Psicofísicos para os Adultos Jovens (GA) na Tarefa de Percepção de Distância em uma Perspectiva Dinâmica sob Privaşão Visual

\begin{tabular}{ccccccc}
\hline Participantes & $1^{\mathrm{a}} \cdot \mathrm{T}$ & $2^{\mathrm{a}} \cdot \mathrm{T}$ & $1^{\mathrm{a}} \cdot \mathrm{T}$ & $2^{\mathrm{a}} \cdot \mathrm{T}$ & $1^{\mathrm{a}} \cdot \mathrm{T}$ & $2^{\mathrm{a}} \cdot \mathrm{T}$ \\
\hline 01 & 1,03 & 0,96 & 0,92 & 1,05 & 0,97 & 0,99 \\
02 & 1,05 & 1,01 & 0,76 & 1,11 & 0,92 & 0,92 \\
03 & 1,03 & 1,08 & 1,16 & 1,19 & 0,98 & 0,99 \\
04 & 1,01 & 0,91 & 1,00 & 1,28 & 0,97 & 0,99 \\
05 & 1,03 & 1,19 & 0,79 & 0,64 & 0,97 & 0,99 \\
06 & 0,89 & 0,99 & 1,25 & 1,08 & 0,97 & 0,99 \\
07 & 0,91 & 1,20 & 0,90 & 0,59 & 0,85 & 0,98 \\
08 & 1,04 & 0,83 & 1,02 & 1,45 & 0,99 & 0,96 \\
09 & 0,95 & 1,08 & 1,07 & 0,87 & 0,98 & 0,99 \\
10 & 0,95 & 0,86 & 0,88 & 0,95 & 0,97 & 0,98 \\
\hline média & 0,99 & 1,01 & 0,97 & 1,02 & 0,96 & 0,98 \\
$d p$ & 0,06 & 0,13 & 0,16 & 0,27 & 0,04 & 0,02 \\
\hline
\end{tabular}

Tabela 2

Valores dos Parâmetros Psicofísicos para Crianças (GC) na Tarefa de Percepção de Distância em uma Perspectiva Dinâmica sob Privação Visual

\begin{tabular}{cccccccc}
\hline & \multicolumn{3}{c}{$n$} & & $k$ & \multicolumn{2}{c}{$r^{2}$} \\
Participantes & $1^{\text {a }} \cdot \mathrm{T}$ & $2^{\mathrm{a}} \cdot \mathrm{T}$ & $1^{\mathrm{a}} \cdot \mathrm{T}$ & $2^{\mathrm{a}} \cdot \mathrm{T}$ & $1^{\mathrm{a}} \cdot \mathrm{T}$ & $2^{\mathrm{a}} \cdot \mathrm{T}$ \\
\hline 01 & 0,09 & 0,98 & 1,00 & 0,68 & 0,87 & 0,99 \\
02 & 0,62 & 1,07 & 2,83 & 0,96 & 0,87 & 0,93 \\
03 & 0,84 & 0,83 & 0,95 & 1,03 & 0,99 & 0,98 \\
04 & 0,81 & 1,20 & 1,21 & 0,61 & 0,87 & 0,92 \\
05 & 1,03 & 1,09 & 0,71 & 0,91 & 0,94 & 0,99 \\
06 & 0,91 & 0,97 & 1,04 & 0,90 & 0,97 & 0,88 \\
07 & 1,05 & 1,02 & 0,62 & 0,77 & 0,97 & 0,87 \\
\hline média & 0,88 & 1,02 & 1,20 & 0,84 & 0,93 & 0,94 \\
$d p$ & 0,15 & 0,12 & 0,75 & 0,15 & 0,05 & 0,05 \\
\hline
\end{tabular}

observar os resultados dos parâmetros psicofísicos (expoente e constante escalar). $\mathrm{O}$ valor do coeficiente de determinação $\left(r^{2}\right)$ foi alto para os dois grupos testados, mostrando um bom relacionamento entre estímulo e resposta (Tabelas 1 e 2).

$\mathrm{Na}$ Figura 4a podemos observar que o GC exibe uma leve diminuição na inclinação da reta $(n=0,88)$ durante a primeira série de tentativa. A segunda tentativa segue um curso linear $(n=1,02)$. O posicionamento da reta logo abaixo da reta de referência indica que as distâncias produzidas embora com incrementos lineares são sempre de magnitudes inferiores à distância testada. Ou seja, as distâncias são subestimadas uma a uma. O grupo adulto, por sua vez, é extremamente acurado, produzindo respostas quase exatas (Figura 4b).
Com a finalidade de verificar se as crianças de fato subestimaram as distâncias, testes $t$-student foram aplicados com o intuito de comparar se os resultados das regressões (intercepto e inclinação da reta) foram diferentes de um ajuste perfeito ( 0 e 1 , respectivamente). Para o GA não houve diferença entre os valores da regressão e os valores do ajuste perfeito. Portanto, o GA foi muito acurado nos julgamentos de distância. Entretanto, para o GC houve diferença entre os valores da regressão e do ajuste perfeito somente na primeira série de tentativas para a inclinação da reta $(t(12)=-3,563 ; p<0,01)$. Para os valores do intercepto houve um efeito marginal $(t(12)=2,689 ; p<$ $0,05)$. Isso indica que as crianças tenderam a subestimar os estímulos na primeira série de tentativas. 


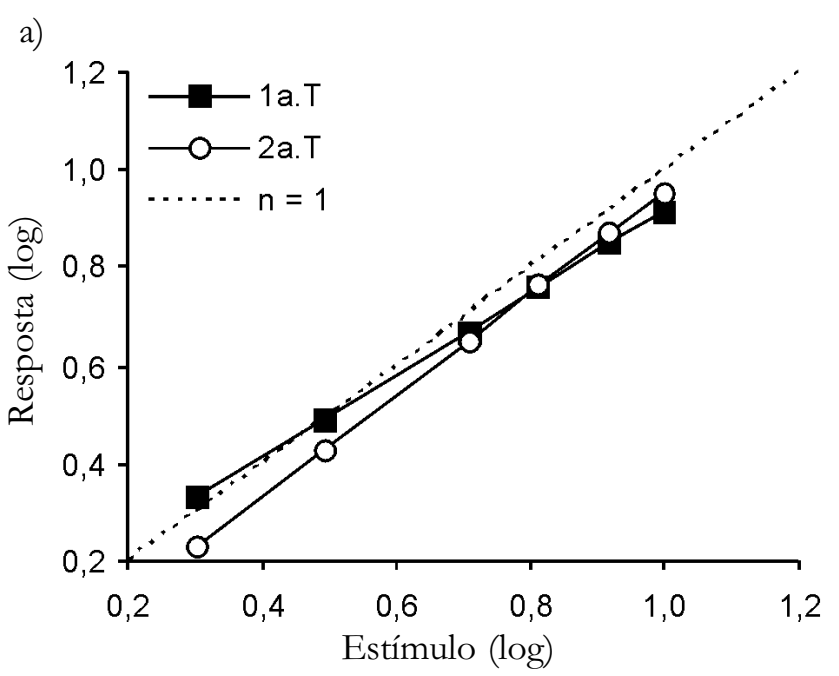

b)

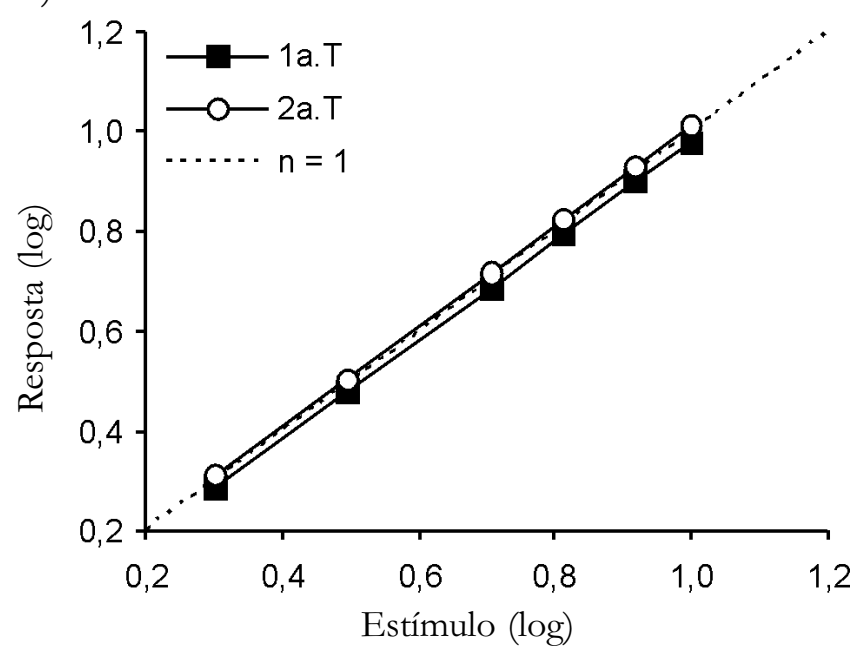

Figura 4. Valores médios do expoente nas duas séries de tentativa para os participantes do grupo criança (a) e grupo adulto (b). As linhas com quadrado representam a primeira tentativa, enquanto que as linhas com círculos a segunda tentativa. A linha sem marcador representa o expoente igual a 1 .

\section{Discussão}

Os resultados obtidos para as variáveis biomecânicas sugerem que as estratégias de controle adotadas entre os dois grupos são as mesmas. Os valores quantitativos são uma evidência disso embora possam ser alteradosdentro de limites permitidos pela coordenação motora sem, entretanto afetar o padrão (i.e., a forma do movimento). As diferenças associadas com a tarefa de produção de distância (i.e., menor PP, DP e VP no estímulo de $2 \mathrm{~m}$ ) podem ser interpretadas como uma adaptação biomecânica decorrente das forças inerciais vencidas durante o início das primeiras passadas e que podem ter influenciado estas variáveis por causa do menor espaço percorrido (i.e., $\pm 2 \mathrm{~m}$ ). Além disso, passadas mais curtas e mais lentas garantem uma maior precisão. Outros componentes do sistema locomotor tomam parte nas adaptações da tarefa como no caso de executar com precisão. McFadyen, Magnan e Boucher (1993), por exemplo, encontraram alterações no momento de força do quadril e joelho quando andando em direção a obstáculos colocados em diferentes distâncias do ponto de partida. Essas alterações do sistema de controle motor foram necessárias para garantir a precisão da locomoção e evitar colisões.

A locomoção (e suas formas diferencias de mobilidade) é consolidada nos primeiros anos de vida e, portanto, faz parte do repertório das habilidades motoras fundamentais (Gallahue \& Ozmun, 1998; Sutherland, 1997). Uma vez que a exploração é uma atividade subordinada à mobilidade (Ex.: marcha), sua evolução pode refletir-se na acurácia em tarefas de percepção de distância (Mauerberg-deCastro \& cols., 2001), na precisão durante a interceptação de objetos em deslocamento (Rodrigues, 1997), na orientação no espaço (Mauerberg-deCastro \& cols., 2001) e no conhecimento sobre dimensões e propriedades do espaço (Daum \& Fahle, 1997). Embora a experiência com a mobilidade tenha sido previamente relacionada com a acurácia perceptiva em estudos clássicos como de Eleanor Gibson (Gibson, 1969), o ganho postural e de controle durante a evolução da marcha logo no início do segundo ano de vida permitem que as crianças realizem tais julgamentos sobre as propriedades do espaço com bastante eficiência. Mesmo em situações onde a postura é atípica, uma vez alcançado um nível de funcionalidade postural, as funções perceptivas podem mostrar-se indiferenciadas quando comparadas com situações normais. De fato, em estudo anterior, observamos que, mesmo na presença de dificuldades de marcha decorrentes de paralisia cerebral, os julgamentos de distância são preservados, inclusive com representações cognitivas acuradas sobre as magnitudes do espaço medidas através de emparelhamento intermodal (Mauerberg \& cols., 1996).

As dimensões testadas no presente estudo foram simples o suficiente para o desempenho invariado entre as crianças na faixa entre cinco e sete anos e os adultos. A sofisticação perceptual é possível na medida em que as crianças ampliam suas experiências locomotoras (Ex.: engatinhar, andar, correr, nadar, mudar de direção e assim por diante). Esta relação entre ação e percepção evidencia a adaptação. Da mesma forma, crianças e adultos também mudam suas estratégias locomotoras em função da experiência e da prática. Por exemplo, no estudo de 
Schuller (2000), atletas demonstraram melhor acurácia em tarefas de produção de distância durante corrida do que seus pares sedentários.

Do ponto de vista ecológico, o conhecimento sobre o espaço e a diversidade de humanos em explorarem-no evidencia a dinâmica estabelecida segundo o contexto. $\mathrm{O}$ contexto do desenvolvimento foi explorado em nosso estudo e seus resultados apontam para respostas uniformes entre os dois grupos. As respostas biomecânicas são relativamente invariantes, exceto na distância inicial. As respostas psicofísicas são dependentes da magnitude do estímulo e não revelam estilos diferenciados entre adultos e crianças.

O fato é que, embora o alvo visualizado por poucos segundos antes do início da tarefa, possa ter contribuído para a acurácia dos grupos, ele não é essencial para o desempenho na navegação pelo ambiente. O estudo de Philbeck e colaboradores (2001) comprova este aspecto onde a resposta de retornar a um ponto de origem após deslocamento com os participantes sob completa privação visual com e sem pré-visualização do alvo não mostra diferenças no desempenho. Ou seja, existe um forte acoplamento entre a representação espacial (não visual) e a ação em si.

Crianças podem utilizar da diversificação de estratégias de exploração do espaço por conta de sua natureza motivacional altamente flutuante. Ou seja, a tarefa de ir e vir de um lugar para o outro geralmente é um processo impregnado de acasos ao longo da rota. Se a rota é curta, é possível que a criança cumpra seu destino. Se a rota é longa, é bem possível que outros assuntos de natureza não controlável num experimento (Ex.: fantasia) possam justificar a possível diversidade exploratória. Curiosidade, atenção e motivação podem juntas afetar ou não o desempenho numa tarefa de simples produção de distância. Nosso estudo impôs certas regras de conduta (Ex.: sistema de roldanas, prática e a noção de participação num jogo) que são desnecessárias para os adultos. Não é impossível que as crianças mantenham-se disciplinadas e concentradas naquilo que se quer que elas façam. Porém, na realidade infantil, é comum que a criança mude de idéia continuamente durante atos exploratórios sobre um ambiente que ainda está por ser dominado em relação ao potencial de ação emergente. Este é um problema ecológico a ser avaliado. Adultos podem manter o curso de ação relativamente inalterado ao longo de um trajeto. Este é o contexto exploratório (ou ecológico) do adulto que vai de um lado para o outro. Ambos, adultos e crianças podem chegar ao mesmo tempo e com a mesma precisão ao local de destino. É o processo de ir até o destino que pode ser diferenciado se as condições naturais forem mantidas. Infelizmente, se o objetivo é medir acurácia e mecanismos perceptuais específicos, não é facil controlar as variáveis presentes em ambientes naturais.

O presente estudo apresenta algumas considerações sobre a natureza da ação e percepção durante julgamentos de distância. Crianças e adultos mantém, quando engajados numa tarefa específica, um desempenho motor semelhante e alterado somente por dimensões física do percurso (i.e., distâncias curtas). Durante a produção de distância, a tendência à subconstância pelas crianças, embora não significativa, ainda é detectável e corrobora resultados de outros estudos, ainda que sob outras condições experimentais.

Futuros estudos devem explorar os mecanismos de percepção do espaço em contextos dinâmicos e relacionálos com o nível evolutivo da mobilidade, no caso, a locomoção. Isto permitiria observar o quão estreitamente estão relacionadas a emergência das noções sobre o espaço e a emergência da ação. Ainda, a sofisticação de percursos e ampliação de dimensões de distâncias podem testar a resistência à deterioração da acurácia perceptiva de crianças por conta de fatores motivacionais, de memória, atenção ou outros.

Em resumo, pode ser concluído que crianças da faixa etária considerada no presente estudo demonstram—em tarefas não usuais (Ex.: locomoção sem visão) adaptabilidade e capacidade de orientar-se no espaço utilizando apenas da sensibilidade háptica e provavelmente da imagem mental. Esta última provavelmente é construída da observação feita antes da realização da tarefa sem informação visual. Além disso, os ajustes biomecânicos observados são similares entre estas crianças e os adultos.

\section{Referências}

Cohen, M. E., Hoffman, H. S., Kelley, N. E. \& Anday, E. K. (1988). A failure to observe habituation in the human neonate. Infant Behavior and Development, 11, 297-304.

Corlett, J. T., Anton, J., Kozub, S. \& Tardif, M. (1989). Is locomotor distance estimation guided by visual imagery? Perceptual and Motor Skills, 69, 1267-1272.

Corlett, J. T., Patla, A. E. \& Williams, J. G. (1985). Locomotor estimation of distance after visual scanning by children and adults. Perception, 14, 257-263.

DaSilva, J. A. (1983). Scales for measuring subjective distance in children and adults in a large open field. Journal of Psychology, 113, 221-229.

DaSilva, J. A. \& Macedo, L. (1983). A função potência na percepção: Significado e procedimentos de cálculo do expoente. Arquivos Brasileiros de Psicologia, 34, 27-45.

DaSilva, J. A., Santos, R. A. \& Silva, C. B. (1983). Análise psicofísica do espaço visual. Arquivos Brasileiros de Psicologia, 35, 3-53.

Elliott, D. (1987). The influence of walking speed and prior practice on locomotor distance estimation. Journal of Motor Behavior, 19, 476-485.

Farrell, M. J. \& Thomson, J. A. (1999). On-line updating of spatial information during locomotion without vision. Journal of Motor Behavior, $31,39-53$. 
Fahle, M. \& Daum, I. (1997). Visual learning and memory as functions of age. Neuropsychology, 35, 1583-1589.

Gallahue, D. L. \& Ozmun, J. C. (1998). Understanding motor development: Infants, cbildren, adolescents, adults. Boston: McGraw-Hill.

Gibson, E. J. (1969). Principles of perceptual learning and development. New York: Meredith Corporation.

Mauerberg, E., Schuller, J. \& Moraes, R. (1996). Perception of distance and matching distance with sound by individuals with cerebral palsy: A psychophysical analysis (pp. 68-69) Third Paraolympic Congress, Atlanta.

Mauerberg-deCastro, E., Moraes, R., Paioli, C., Campos, C., DePaula, A. \& Palla, A. C. (2001). Efeitos da restrição visual e da complexidade de rotas em tarefas de orientação espacial em adultos portadores de deficiência mental. Motriz, 7, 7-16.

McFayden, B. J., Magnan, G. A. \& Boucher, J. P. (1993). Anticipatory locomotor adjustments for avoiding visible, fixed obstacles of varying proximity. Human Movement Science, 12, 259-272.

Moraes, R. (1999). Efeitos do envelhecimento nas habilidades de andar para frente, andar para trás, sentar e levantar. Dissertação de Mestrado não-publicada, Curso de Pós-graduação em Ciências da Motricidade, Universidade Estadual Paulista. Rio Claro, SP.

Okabe, A., Aoki, K., Hamamoto, W. (1986). Distance and direction judgment in a large-scale natural environment: Effects of a slope and winding trail. Environment and Behavior, 18, 755-772.

Philbeck, J. W., Klatzky, R. L., Behrmann, M., Loomis, J. M. \& Goodridge, J. (2001). Active control of locomotion facilities nonvisual navigation. Journal of Experimental Psychology: Human Perception and Performance, 27, 141-153.

Rieser, J. J. (1990). Development of perceptual-motor control while walking without vision: The calibration of perception and action. Em H. Bloch \& B. I. Bertenthal (Orgs.), Sensory-motor organizations and development in infancy and early childhood (pp. 79-108). Netherlands: Kluwer.
Rieser, J. J., Ashmead, D. H. \& Taylor, C. R. (1990). Visual perception and the guidance of locomotion without vision to previously seen targets. Perception, 19, 675-689.

Rieser, J. J., Guth, D. A. \& Weatherford, D. L. (1987). Mentally retarded and nonretarded adults' sensitivity to spatial structure. American Journal of Mental Deficiency, 91, 379-391.

Rieser, J. J. \& Rider, E. A. (1991). Young children's spatial orientation with respect to multiple targets when walking without vision. Developmental Psychology, 27, 97-107.

Rodrigues, S. T. (1997). O timing visual e a informação de tempo para contato. Em A. M. Pellegrini (Org.), Coletannea de estudos: Comportamento motor $I$ (pp.97-108). São Paulo: Movimento.

Schuller, J. (2000). A percep̣cão de distância em perspectiva dinâmica: Restrições do organismo, ambiente e tarefa. Dissertação de Mestrado não-publicada, Curso de pós-graduação em Ciências da Motricidade, Universidade Estadual Paulista Júlio de Mesquita Filho. Rio Claro, SP.

Stevens, S. S. (1975). Psychophysics: Introduction to its perceptual, neural and social prospects. New York: Wiley Interscience.

Sutherland, D. (1997). The development of mature gait. Gait e Posture, 6, 163-170.

Warren, W. H. J. \& Whang, S. (1987). Visual guidance of walking through apertures: Body-scaled information for affordances. Journal of Experimental Psychology: Human Perception and Performance, 13, 371-383.

Sobre os autores

Eliane Mauerberg-deCastro é Professor Adjunto e Doutora em Ciências (área de psicobiologia) na Universidade de São Paulo, Ribeirão Preto. É filiada ao Laboratório da Ação e Percepção do Departamento de Educação Física da Universidade Estadual Paulista, Rio Claro.

Renato Moraes é Mestre em Ciências da Motricidade pela UNESP, Rio Claro. É Doutorando na área de Biomecânica (sob apoio da CAPES) na University of Waterloo, Faculty of Applied Health Science, Departament of Kinesiology Gait and Posture Laboratory. 